\title{
Exploring Factors Impacting Paths of Left-Turning Vehicles from Minor Road Approach at Unsignalized Intersections
}

\author{
Guoqiang Zhang, ${ }^{1,2}$ Yuli Qi, ${ }^{3}$ and Jun Chen ${ }^{1,2}$ \\ ${ }^{1}$ School of Transportation, Southeast University, Nanjing 210096, China \\ ${ }^{2}$ Jiangsu Province Collaborative Innovation Center of Modern Urban Traffic Technologies, Nanjing 210096, China \\ ${ }^{3}$ Shanghai Municipal Engineering Design Institute (Group) CO., Ltd., The Seventh Institute, Qingdao 266071, China
}

Correspondence should be addressed to Guoqiang Zhang; guoqiang.zhang@163.com

Received 27 December 2015; Accepted 13 March 2016

Academic Editor: Francesco Braghin

Copyright (C) 2016 Guoqiang Zhang et al. This is an open access article distributed under the Creative Commons Attribution License, which permits unrestricted use, distribution, and reproduction in any medium, provided the original work is properly cited.

\begin{abstract}
At unsignalized intersections, left-turning vehicles from minor road approach are more likely to be involved in traffic conflicts and traffic crashes and are one of the most leading factors impacting traffic efficiency and capacity. The authors of the paper observed that some drivers behaved illegally and dangerously while performing left turns from minor road approach, resulting in abnormal trajectories at unsignalized intersections. By applying binary logistic analysis, a probability prediction model was developed to explore various factors affecting probability of normal path taken by drivers while turning left from minor road approach. Based upon the model, measures such as lowering running speeds of vehicles on major road or minor road and adding more lanes on minor road can be used to encourage more drivers to take normal vehicle paths, which is helpful for the improvement of traffic safety, efficiency, and capacity. Results of the paper can be used for the guidance of design and management of unsignalized intersections.
\end{abstract}

\section{Introduction}

The most complex locations within a highway system are intersections [1,2]. Traffic conflicts are easily generated in intersections. This makes many intersections be accident black points, where traffic accidents are more likely to occur than anywhere else on the road. Intersections constitute only a small part of the overall highway system but intersection related crashes constitute more than 50 percent of all crashes in urban areas and over 30 percent in rural areas [3]. Although signalized intersections have been constructed widely, many unsignalized intersections are prevalent in both urban and rural areas.

Vehicular interactions at unsignalized intersections are highly complex. Every driver must find a safe moment for moving into the intersection area, observing current traffic, traffic sign, and pertinent regulations. Drivers intending to enter an intersection are presented with a series of gaps (time headways) in a conflicting traffic stream, which they have to cross and/or merge into. A driver has to accept a gap in the conflicting stream and this decision is influenced by complicated behavioral considerations. Each driver makes individual decisions about when, where, and how to complete the required maneuver, subject to his perceptions of distances, velocities, and own car's performance [4].

During the past several decades, many researchers have studied drivers' behaviors at unsignalized intersections and by observing how they accept gaps in conflicting traffic stream, they have developed various gap acceptance models and theories [5-12]. Based upon the models, it was possible to analyze capacity, control delay, and level of service for vehicles at unsignalized intersections [13].

Sinha and Tomiak [7] introduced a new term "section gap" to define the time intervals measured. The study revealed that section gap acceptance varies with the type of maneuver and the vehicle type. Radwan and Sinha [8] modeled the cumulative probability of accepting the gaps of varying lengths using logit analysis. Madanat et al. [9] used logit modeling techniques to develop gap acceptance functions at a stop 
controlled intersection and used stochastic queuing theory to evaluate the intersection's performance. Pollatschek et al. [10] presented a microscopic decision model for driver gap acceptance behavior when waiting at an unsignalized intersection on minor road approach. Davis and Swenson [11] used logit modeling for studying drivers' left turn gap acceptance. The study revealed that the probability of a driver accepting a gap of a given duration increased as the speed of the oncoming vehicle increased. Guo and Lin [12] designed a survey designed method of rejected and accepted gaps. Four methods for calculating critical gap were proposed.

In the last decade, dangerous behaviors and attitudes of drivers have also received some attention [14-18]. Kaysi [14] hypothesized a behavioral framework that enables the analysis of drivers' attitudes at unsignalized intersections based on the driver's characteristics, his past choices, and the actual conditions. Shinar and Compton [16] investigated three types of behavior that are commonly characterized as "aggressive driving," namely, honking, cutting across one or more lanes in front of other vehicles, and passing on the shoulders. Liu and Lee [17] highlighted a significant parameter in aggressive driving: that of car-phone use during critical driving maneuvers. Kaysi and Abbany [18] modelled aggressive driver behavior at unsignalized intersections and concluded that age, car performance, and average speed on the major road were the major determinants of aggressive behavior.

The researches discussed above were helpful for us to understand the performance of unsignalized intersections. However, it should be noticed that some important elements have been ignored. In fact, when drivers decide to accept a gap in conflicting traffic streams, they have to plan a path to cross the road. Patterns of vehicle path (trajectory) represent drivers' behavior and are closely related to traffic safety. Literature review shows that no studies have focused on vehicle path at unsignalized intersections.

Some researchers noticed the impact of intersection layout on vehicle paths and studied variations in vehicle turning trajectories $[19,20]$. The studies were important for the understanding of vehicle paths at intersections. However, at unsignalized intersections, trajectories of turning vehicles are rather complicated. They are affected by many different factors. Besides geometric design, factors such as time headway of vehicles on major road and speeds of turning vehicles also influence paths of turning vehicles. No research has been carried out to study the impacts of the various factors.

At unsignalized intersections, compared with other vehicles, left-turning vehicles from minor road approach are faced with greater danger. They must keep a good watch for potential conflicts while planning left-turning trajectory. This is especially true in developing countries where illegal and dangerous behaviors are rather prevalent. The primary objective for this study is to explore various factors impacting paths of left-turning vehicles from minor road approach at unsignalized intersection. The study will help engineers have a better understanding of how drivers behave while making left turns.

\section{Data Collection and Method}

2.1. Data Collection. In China, at stop controlled intersections, vehicles from minor road approach do not stop before the stop line when there are no conflicting vehicles on major roads. Thus, stop controlled intersections are very similar to yield controlled intersections. Therefore, the research did not distinguish between stop controlled intersection and yield controlled intersection.

Many factors may impact trajectory of left-turning vehicles and they could be classified into three groups: characteristics of roads and other facilities, characteristics of vehicles, and characteristics of drivers. Because impacting factors were both numerous and confounding, limits were set on scope of the research so that the study could be carried out and important factors could be explored.

The research focused upon the unsignalized intersections whose major road and minor road could be easily identified and whose control devices such as stop signs or yield signs were well maintained. Deformity intersections were excluded from the research because trajectories of vehicles at the intersections were quite unique and the patterns of vehicle paths were not easy to recognize.

It was impossible to acquire information about characteristics of drivers such as age, gender, education level, years of driving experience, accident involvement, and attitudes when they were making left turns from minor road approach. For this reason, impacts of driver characteristics were excluded from the research.

Characteristics of roads and other facilities include many factors that may have more or less impact upon vehicle path. Among them, number of lanes on the road was selected for analysis, because it represented some of the most influential characteristics of roads such as width and capacity.

Characteristics of vehicles include the most complicated and dynamic information. For example, hundreds and thousands of vehicles may have gone through an intersection in a short period of time during peak hours and traffic situations may be changing all the time. Meanwhile, there are countless factors that may impact driver's behavior and vehicle path. In order to facilitate the research, information concerning vehicle characteristics was selected and simplified.

In China, when traffic volume of bicycles, motorcycles, and pedestrians is not very high, it is common practice for them to yield the right of way to automobiles. Therefore, their influence upon left-turning vehicle path is negligible. For this reason, the research did not take their impact into consideration.

When vehicles on minor road approach turn left, there are many traffic streams that may be in conflict with them. Nevertheless, it is the eastbound through traffic on major road that constitutes the main threat and exerts the most important influence upon trajectories of left-turning vehicles. Two factors concerning through vehicles on major road were selected for analysis. They were time headway of vehicles on major road and running speed of vehicle on major road. Time headway of vehicles on major road was defined as the time headway of vehicles on major road on the near side of minor road approach when vehicle on minor road approach was 
passing the stop line and trying to turn left. Running speed of vehicle on major road was defined as the running speed of the first vehicle before the conflict area on major road on the near side of minor road approach at the moment when vehicle on minor road approach was passing the stop line and trying to turn left.

As for characteristics of vehicles making left turns from minor road approach, two factors were selected and they were type of left-turning vehicle and running speed of leftturning vehicle. Left-turning vehicles were classified into two types: small size vehicles and other vehicles. Running speed of left-turning vehicle was defined as the running speed of leftturning vehicle at the moment when it was passing the stop line on minor road and was trying to turn left.

To control for the impacts of various confounding factors that affect paths of left-turning vehicles from minor road approach, the following criteria were applied in the site selection process:

(1) The selected intersection should be in territory of flat terrain. Vehicle trajectories at intersections are strongly influenced by various types of terrain. The influence of terrain is rather complicated and is excluded from the research.

(2) The selected intersection should be in the suburbs or in rural areas where bicycles, motorcycles, and pedestrians were seldom observed. When there were only a small number of bicycles, motorcycles, or pedestrians, their influence upon vehicle path could be disregarded.

(3) Except through vehicles on major road, the selected intersection should have very few vehicles to interfere with left-turning vehicles from minor road approach. Unsignalized intersections that have a lot of vehicles turning left from the major road approaches or making through movements on minor road should be excluded.

(4) The selected intersection should be isolated. According to Highway Capacity Manual, an intersection can be regarded as isolated when the nearest intersection is more than 5000 feet (1524 meters) away [13]. The value was adopted in the selection of isolated intersections. Traffic flow at such places is effectively random, which meets the requirement of the research.

(5) The selected intersection should have no access connections in its functional area. The functional area extends both upstream and downstream from the physical intersection area and includes the longitudinal limits of auxiliary lanes. This is the area where drivers are responding to the intersection, decelerating, and maneuvering into the appropriate lane to stop or complete a turn. Access connections in the functional area can cause various traffic conflicts that hamper the research.

(6) Traffic volumes of the selected intersection should be moderate. When traffic volumes of unsignalized intersection are low, influence of vehicles on major road will be too small to be analyzed statistically. On the other hand, when traffic volumes are high, it will be difficult for vehicles from minor road approach to turn left without stopping before the stop line.

As far as the objective of this study is concerned, $\mathrm{T}$ shaped intersections were superior to other types of intersections and were given special priority in the site selection process. The reasons for this are as follows. Firstly, $\mathrm{T}$ shaped intersections are found abundantly in roadway systems and are representative of unsignalized intersections; secondly, at $\mathrm{T}$ shaped intersections, minor road was connected to major road at right angles, which is helpful for vehicles on minor road approach to make left-turn or right-turn movements; finally, compared with other types of unsignalized intersections, $\mathrm{T}$ shaped intersections usually do not have a lot of vehicles to interfere with left-turning vehicles from minor road approach except through vehicles on major road. For example, at crisscrossing road intersections, left-turning vehicles from minor road approach are sometimes impacted by vehicles making through movements on minor road in the opposite direction. $T$ shaped intersection does not have this kind of impact which, as discussed above, was excluded from the research.

The research team did a lot of field surveys in Jiangsu Province, a populous and economically developed region in the east of China. After comprehensive analysis, two unsignalized intersections were selected. They were $\mathrm{T}$ shaped intersections with typical geometric designs as shown in Figure 1. The major roads of the selected intersections were provincial trunk roads and the minor roads were country roads. Both of them had four lanes on the major road with two lanes in each direction. One of them had four lanes on the minor road with an exclusive left-turn lane and an exclusive right-turn lane on the approach (see Figure 1(a)). The other intersection had two lanes on the minor road with one lane on the approach shared by left turns and right turns (see Figure 1(b)).

There were tall buildings in the vicinity of the intersections, from the top of which digital video cameras were used to record vehicle movements at the intersections. Special marks were painted on the roads so that it was possible to get the time when a vehicle passed a marked point on the roads from recorded video. Time headway and running speed were then calculated.

The research team played digital video files over and over again and studied every vehicle making left turns from minor road approach. In all, 184 vehicles were found to make left turns from minor road approach without stopping. Data concerning the vehicles, their trajectories, and impacting factors were collected and analyzed.

After exploring various left-turning vehicle paths from video records, six types of vehicle paths were identified. They were defined as type 1 , type 2 , type 3 , type 4 , type 5 , and type 6 left-turning vehicle paths, respectively, based upon characteristics of left-turning vehicle trajectory. Their definitions are illustrated in Figure 1. Their characteristics are briefly described as follows. 


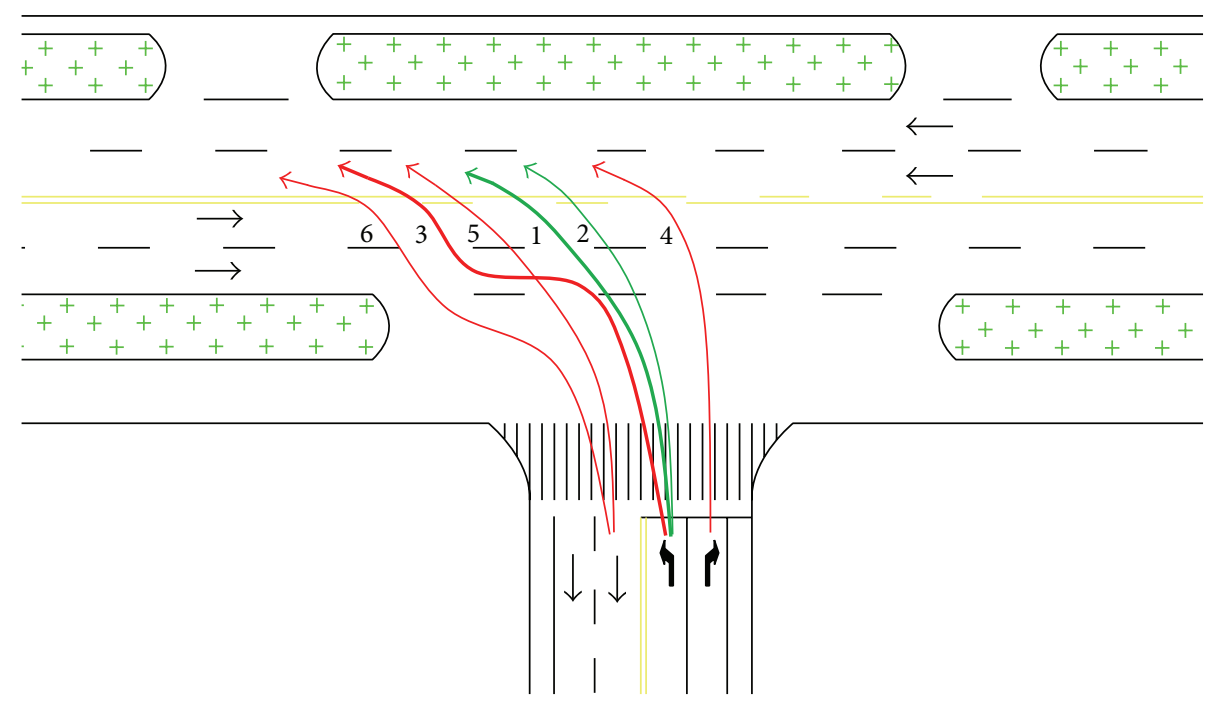

(a)

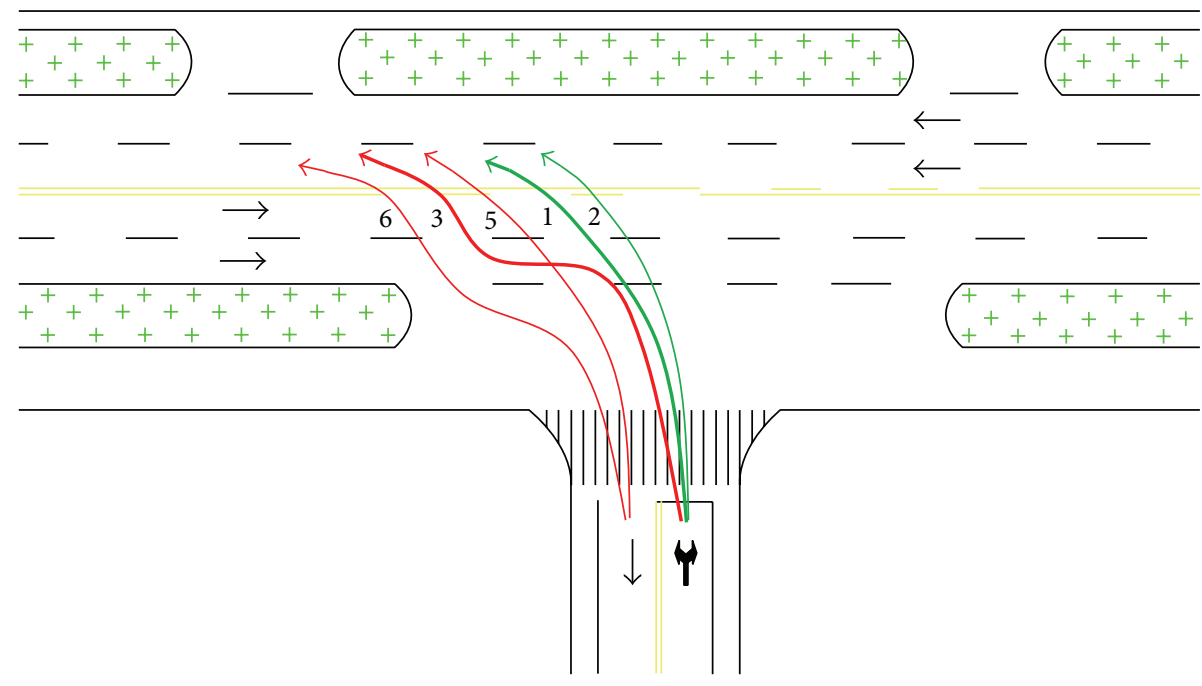

(b)

Figure 1: Paths of left-turning vehicles at unsignalized intersections. (a) Intersection with four lanes on the minor road. (b) Intersection with two lanes on the minor road.

Both type 1 and type 2 left-turning vehicle paths were normal paths which represented drivers' legal and safe behaviors. When drivers took such paths, time headway of vehicles on major road was long enough for left-turning maneuvers. Leftturning vehicles started from a lane on minor road approach and moved fast upward and leftward. After crossing two lanes on major road, left-turning vehicles merged quickly with major road vehicles in the opposite direction. Although the two types of left-turning vehicle trajectories were very similar, there was some slight difference. Compared with type 2, type 1 left-turning vehicle path had a larger turning radius, which enabled left-turn movements to be more fast and comfortable. When left-turning vehicles were affected by bicycles or pedestrians, drivers were forced to slow down and take a more sharp turn and, in that way, they took a type 2 vehicle path. 107 (58.2\%) vehicle paths were found to be type 1 and only $18(9.8 \%)$ paths were found to be type 2 .

Type 3 , type 4 , type 5 , and type 6 vehicle paths were abnormal and they represented drivers' illegal and dangerous behaviors when performing left turns. When time headways on major road were too short to be accepted, drivers would take type 3 paths. Under such circumstance, left-turning vehicles moved leftward slowly near the through traffic stream on major road until there was a gap in the traffic, which was big enough to be used by left-turning vehicles. So, vehicle paths of type 3 were seriously lengthened compared with type 1 paths. In the left-turning process, left-turning vehicles moved near the through traffic in the opposite direction, causing great danger to vehicles on major road. Type 4 paths were very similar to type 2 paths, except that 
type 4 started from the exclusive right-turn lane. Type 5 was similar to type 1 and type 6 was similar to type 3, except that both of them started from the wrong place, which was designed for traffic exiting from the intersections. When a left-turning vehicle was blocked by other vehicles in front of it, the driver sometimes sought to avoid blockage by moving to other lanes illegally and, in this way, type 4 , type 5 , or type 6 vehicle paths were taken by impetuous drivers. It was found that $42(22.8 \%), 12(6.5 \%), 3(1.6 \%)$, and $2(1.1 \%)$ vehicle paths were type 3 , type 4 , type 5 , and type 6 , respectively.

Later on, data concerning type 2 , type 4 , type 5 , and type 6 left-turning vehicle paths were excluded from modelling. It was done under the following considerations: these types of vehicle paths were taken under the impacts of some stochastic factors such as the random appearance of pedestrians or bicycles or driver's rash behavior, which were very difficult to explore; preliminary analysis of data indicated that the types discarded accounted for only a small portion of the total leftturning vehicle paths (19\%); therefore, exclusion of the data did not affect data analysis results too much. After exclusion of these vehicle paths, 149 paths were used in modelling and they were classified as normal (type 1) and abnormal (type 3 ).

2.2. Statistical Method. Linear regression models that have been used in transportation research have the assumption that the response variable is continuous. However, in cases where the response (dependent) variable is not continuous, discrete outcome models should be applied. When there are two discrete outcomes, binary logistic regression models can be applied. Like linear regression, the goal of logistic regression is to identify the best fitting model that describes the relationship between a binary dependent variable and a set of independent or explanatory variables. In contrast to linear regression, the dependent variable is the population proportion or probability $(P)$ that the resulting outcome is equal to 1 . It is important to note that the parameters obtained for the independent variable can be used to estimate odds ratios for each of the independent variables in the model. General form of the binary logistic regression is as follows:

$$
\begin{gathered}
\log \left(\frac{P\left(Y=1 \mid X_{1}, X_{2}, \ldots, X_{p}\right)}{1-P\left(Y=1 \mid X_{1}, X_{2}, \ldots, X_{p}\right)}\right) \\
=\alpha+\beta_{1} X_{1}+\beta_{2} X_{2}+\cdots+\beta_{p} X_{p},
\end{gathered}
$$

where $Y$ is dichotomous dependent variable; $X_{i}$ are independent variables $(i=1,2, \ldots, p) ; \beta_{i}$ are logistic regression coefficients $(i=1,2, \ldots, p)$; and $\alpha$ is intercept term.

The values of the intercept term and the regression coefficient for each independent variable are estimated by the Maximum Likelihood Estimation (MLE) method. The goodness of fit of the model can be assessed with the likelihood ratio test. The likelihood ratio test uses the ratio of the maximized value of the likelihood function for the full model $\left(L_{f}\right)$ over the maximized value of the likelihood function for the simpler model $\left(L_{0}\right)$. The likelihood ratio test statistic is as follows:

$$
-2 \log \left(\frac{L_{0}}{L_{f}}\right)=-2\left[\log \left(L_{0}\right)-\log \left(L_{f}\right)\right] .
$$

The exponential of the coefficient, $\exp \left(\beta_{i}\right)$, expresses the odds ratio (the ratio of probability of occurrence of an event to the probability of nonoccurrence of an event). More specifically, concerning positive coefficients, for each additional unit increase in the variable $X_{i}$, the odds of $Y=1$ are increased by $100 \cdot\left[\exp \left(\beta_{i}\right)-1\right]$ percent. For negative coefficients, a one-unit increase decreases the odds of $Y=1$ by $100 \cdot\left[1-\exp \left(\beta_{i}\right)\right]$ percent.

\section{Data Analysis Results}

In this study, a probability prediction model for left-turning vehicle path types was developed to explore various factors that contribute to driver's choice of paths at unsignalized intersection while making left turns from minor road approach and to explain how time headway of vehicles on major road impacts driver's behavior along with other confounding factors. The dependent variable of the model is type of left-turning vehicle path with 1 for normal path (type 1 vehicle path) and 0 for abnormal path (type 3 vehicle path). Six independent variables were initially considered. The descriptive statistics of these variables are given in Table 1.

In order to choose the significant variables from the six independent variables listed in Table 1, forward stepwise variable selection method with Likelihood-Ratio removal criterion was used. In the first step, the most significant variable is identified and evaluated for incorporation into the model. If this particular variable satisfies the significance level requirement ( 0.05 in this study) then the variable is accepted. In the second step, the model developed in step 1 is used as the base model and significance values of each of the remaining variables for consideration are determined. The most significant variable is evaluated for incorporation into the model. If the variable meets the significance level requirement, the variable is added to the model. Before moving to the next step, variables entered into the model are evaluated for their significance levels by using the likelihood ratio test. If all variables are significant at the specified significance level ( 0.10 in this study), the next step starts. If the significance value for a variable exceeds the predetermined significance level, the variable is removed from the regression model and the model is reevaluated to determine if any additional variables should be removed from the model. The algorithm continues until eliminations result in a previously evaluated model or none of the variables meet entry or removal criteria.

Two variables were found to be statistically insignificant and were excluded from the model. The best model has four independent variables and the results are given in Table 2. The final equation for probability prediction of normal path of left-turning vehicles is given as follows:

$$
\begin{aligned}
& P(Y=1) \\
& =\frac{\exp \left(5.562-1.924 I-0.188 V_{l}-0.036 V_{m}+0.112 T_{m}\right)}{1+\exp \left(5.562-1.924 I-0.188 V_{l}-0.036 V_{m}+0.112 T_{m}\right)},
\end{aligned}
$$

where $Y$ is type of left-turning vehicle path ( 1 for normal path, 0 for abnormal path); $I$ is the number of lanes on minor road ( 1 for two lanes, 0 for four lanes); $V_{l}$ is the running speed of 
TABLE 1: Descriptive statistics of variables for model development.

\begin{tabular}{|c|c|c|c|c|c|}
\hline Variables & Min & Max & Mean & Standard deviation & Frequency \\
\hline Type of left-turning vehicle path & - & - & - & - & 149 \\
\hline 1 (normal path) & - & - & - & - & $107(71.81 \%)$ \\
\hline 0 (abnormal path) & - & - & - & - & $42(28.19 \%)$ \\
\hline Running speed of left-turning vehicle ( $\mathrm{km} / \mathrm{hour}$ ) & 8.54 & 28.80 & 18.32 & 3.60 & 149 \\
\hline Running speed of vehicle on major road ( $\mathrm{km} / \mathrm{hour}$ ) & 0.00 & 72.00 & 18.21 & 23.60 & 149 \\
\hline Time headway of vehicles on major road (seconds) & 1.00 & 48.64 & 12.15 & 8.96 & 149 \\
\hline Type of left-turning vehicle & - & - & - & - & 149 \\
\hline 1 (small size vehicle) & - & - & - & - & $139(93.33 \%)$ \\
\hline 0 (other vehicles) & - & - & - & - & $10(6.67 \%)$ \\
\hline Time of survey & - & - & - & - & 149 \\
\hline 1 (morning) & - & - & - & - & $131(87.92 \%)$ \\
\hline 0 (afternoon) & - & - & - & - & $18(12.08 \%)$ \\
\hline Number of lanes on minor road & - & - & - & - & 149 \\
\hline 1 (two lanes) & - & - & - & - & $47(31.54 \%)$ \\
\hline 0 (four lanes) & - & - & - & - & $102(68.64 \%)$ \\
\hline
\end{tabular}

TABLE 2: Binary logistic model for path of left-turning vehicles from minor road approach.

\begin{tabular}{lcccccc}
\hline Variables & Coefficient & Standard error & Significance & Exp $(B)$ & $\begin{array}{c}\text { 95\% confidence interval for Exp }(B) \\
\text { Lower bound }\end{array}$ & Upper bound \\
\hline Number of lanes on minor road & -1.924 & 0.646 & 0.003 & 0.146 & 0.041 & 0.518 \\
Running speed of left-turning vehicle & -0.188 & 0.073 & 0.010 & 0.828 & 0.718 & 0.956 \\
Running speed of vehicle on major road & -0.036 & 0.010 & 0.000 & 0.965 & 0.946 & 0.984 \\
Time headway of vehicles on major road & 0.112 & 0.040 & 0.005 & 1.119 & 1.034 & - \\
Constant & 5.562 & 1.779 & 0.002 & 260.352 & - \\
\hline
\end{tabular}

-: not applicable.

left-turning vehicle on minor road approach ( $\mathrm{km} / \mathrm{hour}) ; V_{m}$ is the running speed of vehicle on major road ( $\mathrm{km} /$ hour); and $T_{m}$ is time headway of vehicles on major road (seconds).

Table 2 summarizes the output of binary logistic regression analysis. All of the independent variables selected for the model are statistically significant at 0.05 significance level. Coefficients for these variables can be used to analyze impacts of various factors upon probability of normal vehicle path.

The coefficient for the indicator variable for number of lanes on minor road is negative, implying the fact that probability of normal path is higher at intersection with four lanes on the minor road compared with two lanes on the minor road. The exponential of the coefficient $B$ of this variable (0.149) expresses the odds ratio (the ratio of probability of occurrence of an event to the probability of nonoccurrence of an event). Based on the value, if other factors remain the same, replacing a two-lane minor road with a four-lane minor road will result in $1-0.149=85.1 \%$ increase in the odds that normal vehicle paths are taken by drivers performing leftturning maneuvers from minor road approach. Traditionally, increasing the number of lanes has been used to increase capacity and level of service. However, based on the findings of this study, it can be concluded that the number of lanes on the minor road at unsignalized intersection also has an impact on driver's behavior and therefore influences traffic safety. It can be speculated that the reason for a wider minor road (with more lanes) to have a higher probability of normal left-turning vehicle path can be due to the potentially better sight distance which enables drivers of left-turning vehicles to see and assess the possibility of impending hazards posed by vehicles on major road. It can also be speculated that narrower road (with less lanes) may impair driver's ability to accept a gap in the conflicting traffic stream through which left-turning maneuvers can be performed safely. Besides, narrower two-lane minor road (as shown in Figure 1(b)) does not have an exclusive left-turn lane and can bring about more delay and more uncomfortable experience to drivers of leftturning vehicles when confronted with right-turning vehicles competing for the same lane. Unfavorable road conditions of two-lane minor road may lead to dangerous behaviors such as those indicated by abnormal vehicle path.

The coefficient for running speed of left-turning vehicle is negative, which indicates that probability of normal path decreases with increase of running speed of left-turning vehicle. More specifically, drivers who drive faster on minor road approach before performing left-turning operations are less likely to choose a normal path. The value of the odds ratio (0.828) means that a one-unit increase in running speed of 


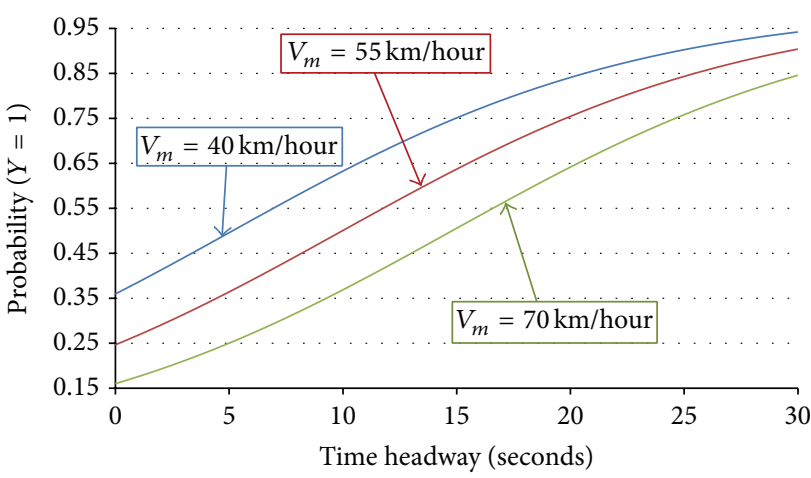

(a)

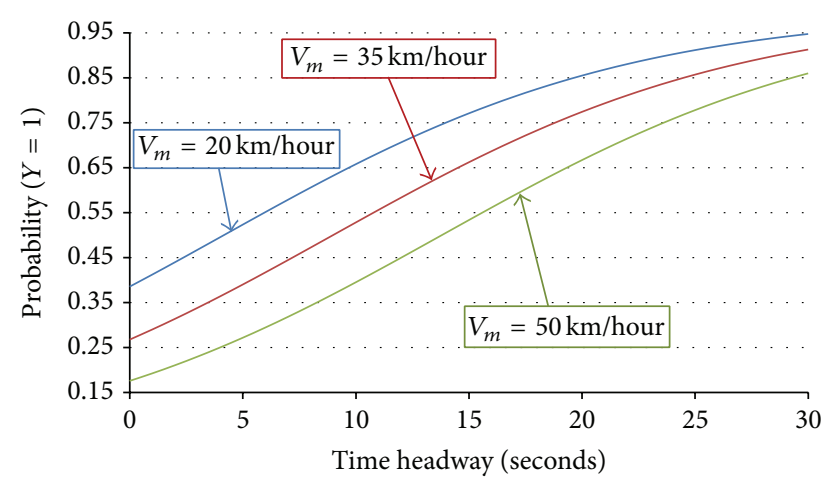

(b)

Figure 2: Probability curves for intersections. (a) Intersection with four lanes on the minor road $\left(V_{l}=25 \mathrm{~km} /\right.$ hour). (b) Intersection with two lanes on the minor road $\left(V_{l}=18 \mathrm{~km} /\right.$ hour$)$.

left-turning vehicle leads to a $17.2 \%$ decrease in the odds of normal path, assuming that all other factors remain constant. A possible explanation is that when a vehicle is running at a faster speed before turning left, it will be difficult for the driver to stop completely before stop line if there are conflicting vehicles on major road. Under such circumstance, drivers tend to take an abnormal path.

Similarly, the coefficient for running speed of vehicle on major road is negative, implying that probability of normal path decreases with increase of running speed of vehicle on major road. More specifically, when vehicles on major road run at a faster speed, vehicle paths of left-turning vehicles from minor road approach are less likely to be normal. The value of the odds ratio $(0.965)$ means that a one-unit increase in running speed of vehicle on major road leads to a $3.5 \%$ decrease in the odds of normal path, assuming that all other factors remain constant. When drivers of vehicles on minor road approach are preparing to turn left, faster moving vehicles on major road pose impending hazards. To avoid potential conflicts and accidents, drivers may tend to take a detoured abnormal path.

Lastly, the coefficient for time headway of vehicles on major road is positive, indicating that probability of normal path increases with increase of time headway of vehicles on major road. Besides, as is known, traffic volume is an inverse proportion function of time headway of vehicles. Therefore, when traffic volume of major road becomes lower, time headway increases and vehicle paths of left-turning vehicles from minor road approach are more likely to be normal. The value of the odds ratio (1.119) means that a one-unit increase in time headway of vehicles on major road leads to a $11.9 \%$ increase in the odds of normal path, assuming that all other factors remain constant. The reasons for such a phenomenon are quite obvious. When time headway of vehicles increases, drivers of vehicles on minor road approach are provided with more opportunity to make a normal left turn without detouring abnormally.

Further analysis indicates that performance of the model is acceptable. For abnormal path $(Y=0)$, percentage of correct predictions is $57.1 \%$. For normal path $(Y=1)$, percentage of correct predictions is $88.8 \%$. The overall percentage of
TABLE 3: Classification table.

\begin{tabular}{|c|c|c|c|}
\hline \multicolumn{3}{|c|}{ Observed Estimated path type } & \multirow{2}{*}{ Percentage of correct predictions } \\
\hline path type & 0 & 1 & \\
\hline 0 & 24 & 18 & $57.1 \%$ \\
\hline 1 & 12 & 95 & $88.8 \%$ \\
\hline $\begin{array}{l}\text { Overall } \\
\text { percentage } \\
\text { of correct } \\
\text { predictions }\end{array}$ & - & - & $79.9 \%$ \\
\hline
\end{tabular}

-: not applicable.

correct predictions is $79.9 \%$. The resulting classification table is provided in Table 3, which implies that predictions of the model are in good agreement with observed facts. Therefore, the model can be used to predict possibility of normal path in various situations.

To better understand influences of various factors, probability curves have been drawn to show how various factors impact probability of normal path while vehicles make left turns from minor road approach by using (3). Probability for normal path is depicted as a function of time headway of vehicles on major road (holding all other explanatory variables at a fixed value). To facilitate analysis of the impact of $V_{m}$ (running speed of vehicle on major road), three probability curves are drawn separately for an intersection, assuming three typical values of $V_{m}$. Figure 2 is provided as an example to demonstrate the shape of the probability curves for intersection with four lanes on the minor road assuming $V_{l}$ (running speed of left-turning vehicle) is $25 \mathrm{~km} /$ hour and intersection with two lanes on the minor road assuming $V_{l}$ is $18 \mathrm{~km} /$ hour.

The curves indicate that probability for normal path increases with the increase of $T_{m}$ (time headway of vehicles on major road). The fact that probability curves with smaller values of $V_{m}$ are above those with larger ones implies that drivers are more likely to take normal path when they are confronted with slower moving vehicles on major road. According to Figure 2(a), for intersection with four lanes on the minor road, assuming $V_{l}$ is $25 \mathrm{~km} /$ hour, if $V_{m}$ is $40 \mathrm{~km} /$ hour, 
probability for normal path becomes higher than probability for abnormal path once $T_{m}$ reaches 5.2 seconds. However, if vehicles on major road are running at a higher speed, such as 55 and $70 \mathrm{~km} /$ hour, longer $T_{m}$ is needed for drivers to prefer normal path. If $V_{m}$ is $55 \mathrm{~km} /$ hour, the critical time headway is 10 seconds. For $70 \mathrm{~km} /$ hour, the critical time headway is 14.9 seconds. Similarly, according to Figure 2(b), for intersection with two lanes on the minor road, assuming $V_{l}$ is $18 \mathrm{~km} /$ hour, if $V_{m}$ is 20,35 , and $50 \mathrm{~km} /$ hour, respectively, the critical time headway is $4.2,9$, and 13.9 seconds.

\section{Discussions}

This study evaluated how various factors affected vehicle paths while drivers were making left turns from minor road approach at unsignalized intersection. Two typical intersections were selected as the field observation sites. Data were collected with the assistance of a video camera. Six types of left-turning vehicle paths were observed. Two of them (type 1 and type 2) were normal paths and the other four types (type 3, type 4 , type 5, and type 6) were abnormal paths. Characteristics of these types of vehicle paths were discussed. Data for type 1 (normal path) and type 3 (abnormal path) vehicle path were used in model calibration.

By applying binary logistic analysis, a probability prediction model was developed to explore all sorts of factors that affect the probability of normal path. It was found that number of lanes on minor road, running speed of left-turning vehicle, running speed of vehicle on major road, and time headway of vehicles on major road significantly affected probability of normal path. Performance of the model is acceptable and the overall percentage of correct predictions is $79.9 \%$.

Based upon the probability prediction model for normal path, replacing a two-lane minor road with a four-lane minor road will result in $85.1 \%$ increase in the odds of normal path, assuming that all other factors remain constant. A one-unit increase in running speed of left-turning vehicle leads to a $17.2 \%$ decrease in the odds of normal path and a one-unit increase in running speed of vehicle on major road leads to a $3.5 \%$ decrease in the odds of normal path. In addition, a oneunit increase in time headway of vehicles on major road leads to a $11.9 \%$ increase in the odds of normal path.

Probability curves have been drawn to show relationship between probability of normal path and time headway of vehicles on major road. The curves can be used to visually display how various factors impact probability of normal path. According to the probability curves, there is a critical value for time headway of vehicles on major road. When time headway reaches or exceeds such a value, probability for normal path becomes higher than probability for abnormal path.

Abnormal paths of left-turning vehicles are serious illegal behaviors which affect vehicles on major road and interfere with both efficiency and safety of unsignalized intersections. The results of this study indicate that, except for education and enforcement, engineering measures can also be used to affect drivers' behaviors and improve traffic safety and efficiency. For example, lowering running speeds of vehicles while they are approaching intersections or adding more lanes on minor road can be used to encourage more drivers to take normal vehicle path, which will in turn lead to improvement of traffic safety and efficiency at unsignalized intersections.

\section{Conclusions}

The results of this study can assist researchers and practitioners in further understanding impacting factors underlying vehicle driver's abnormal behaviors when driving at unsignalized intersections. The prediction model can be used to quantify the impacts of various explanatory variables on vehicle path of left-turning vehicles from minor road. Designers can also use the model to evaluate how geometric designs such as number of lanes and design speeds affect vehicle movements at unsignalized intersections. The research results have the propensity to help transportation decision-makers develop technical guidelines governing the design and management of unsignalized intersections.

Improvement needs to be made in order to rectify the limitations in this study. Except for paths of left-turning vehicles from minor road, other vehicle paths such as those of left-turning and right-turning vehicles from major road should also be explored. This can lead to more comprehensive understanding of how vehicles from various approaches interact with each other at unsignalized intersections, based upon which effective measures can be taken to influence driver's behavior in a positive way.

\section{Competing Interests}

The authors declare that there are no competing interests regarding the publication of this paper.

\section{Acknowledgments}

This research was jointly supported by Humanity and Social Science Youth Foundation of Ministry of Education of China (Project no.: 10YJCZH214) and National Natural Science Foundation of China (Project no.: 51278103). Their assistance is greatly appreciated.

\section{References}

[1] S.-S. Li, D.-L. Qian, and Y. Luo, "Microscopic dynamic simulation model for pedestrian at signalized intersection," Journal of Central South University, vol. 19, no. 11, pp. 3351-3362, 2012.

[2] Z.-B. He, S.-F. Ma, and W. Guan, "Delays caused by motorized vehicles unable to clear intersections in China: graphical analysis," Journal of Central South University, vol. 20, no. 9, pp. 26142624, 2013.

[3] Transportation Research Board, Guidance for Implementation of the AASHTO Strategic Highway Safety Plan, Volume 5: A Guide for Addressing Unsignalized Intersection Collisions, Transportation Research Board, Washington, DC, USA, 2003.

[4] Transportation Research Board, Review of International Practices Used to Evaluate Unsignalized Intersections, Transportation Research Board, Washington, DC, USA, 1997. 
[5] P. Solberg and J. C. Oppenlander, "Lag and gap acceptances at stop controlled intersections," Highway Research Record, vol. 118, pp. 48-67, 1966.

[6] R. Ashworth and B. D. Green, "Gap acceptance at an uncontrolled intersection," Traffic Engineering and Control, vol. 7, no. 11, pp. 676-678, 1966.

[7] K. C. Sinha and W. W. Tomiak, "Section gap acceptance phenomenon at stop-controlled intersections," Traffic Engineering and Control, vol. 41, no. 7, pp. 28-33, 1971.

[8] A. E. Radwan and K. C. Sinha, "Gap acceptance and delay at stop controlled intersections on multilane divided highways," Journal of Institute of Transportation Engineers, vol. 50, no. 3, pp. 38-44, 1980.

[9] S. M. Madanat, M. J. Cassidy, and M.-H. Wang, "Probabilistic delay model at stop controlled intersection," Journal of Transportation Engineering, vol. 120, no. 1, pp. 21-36, 1994.

[10] M. A. Pollatschek, A. Polus, and M. Livneh, "A decision model for gap acceptance and capacity at intersections," Transportation Research Part B: Methodological, vol. 36, no. 7, pp. 649-663, 2002.

[11] G. A. Davis and T. Swenson, "Field study of gap acceptance by left-turning drivers," Transportation Research Record, vol. 1899, pp. 71-75, 2004.

[12] R.-J. Guo and B.-L. Lin, "Gap acceptance at priority-controlled intersections," Journal of Transportation Engineering, vol. 137, no. 4, pp. 269-276, 2011.

[13] Transportation Research Board, Highway Capacity Manual, Transportation Research Board, Washington, DC, USA, 2010.

[14] I. Kaysi, The Leading Edge in Travel Behavior Research, Pergamon Press, Oxford, UK, 2001.

[15] D. E. Miles and G. L. Johnson, "Aggressive driving behaviors: are there psychological and attitudinal predictors?" Transportation Research Part F: Traffic Psychology and Behaviour, vol. 6, no. 2, pp. 147-161, 2003.

[16] D. Shinar and R. Compton, "Aggressive driving: an observational study of driver, vehicle, and situational variables," Accident Analysis and Prevention, vol. 36, no. 3, pp. 429-437, 2004.

[17] B.-S. Liu and Y.-H. Lee, "Effects of car-phone use and aggressive disposition during critical driving maneuvers," Transportation Research-Part F: Traffic Psychology and Behaviour, vol. 8, no. 4-5, pp. 369-482, 2005.

[18] I. A. Kaysi and A. S. Abbany, "Modeling aggressive driver behavior at unsignalized intersections," Accident Analysis and Prevention, vol. 39, no. 4, pp. 671-678, 2007.

[19] W. K. M. Alhajyaseen, M. Asano, H. Nakamura, and D. M. Tan, "Stochastic approach for modeling the effects of intersection geometry on turning vehicle paths," Transportation Research Part C: Emerging Technologies, vol. 32, pp. 179-192, 2013.

[20] D. Tan, W. Alhajyaseen, M. Asano, and H. Nakamura, "Development of microscopic traffic simulation model for safety assessment at signalized intersections," Transportation Research Record, vol. 2316, pp. 122-131, 2012. 


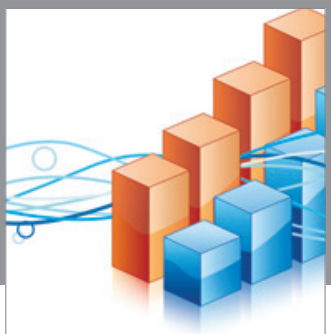

Advances in

Operations Research

vatem alat4

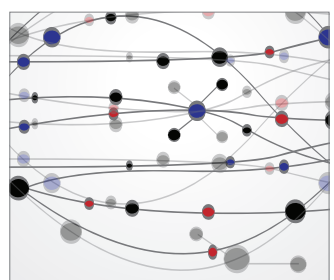

\section{The Scientific} World Journal
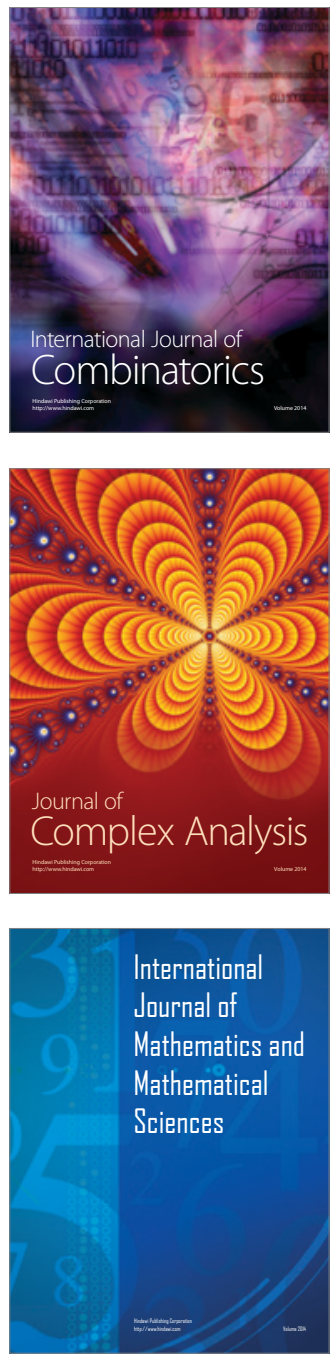
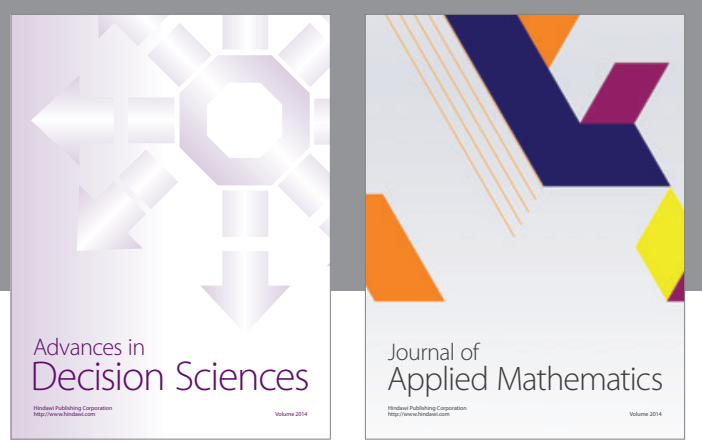

Algebra

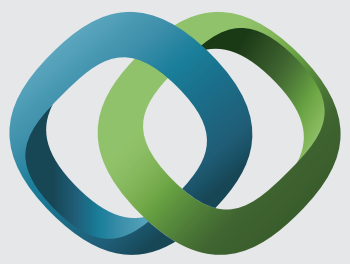

\section{Hindawi}

Submit your manuscripts at

http://www.hindawi.com
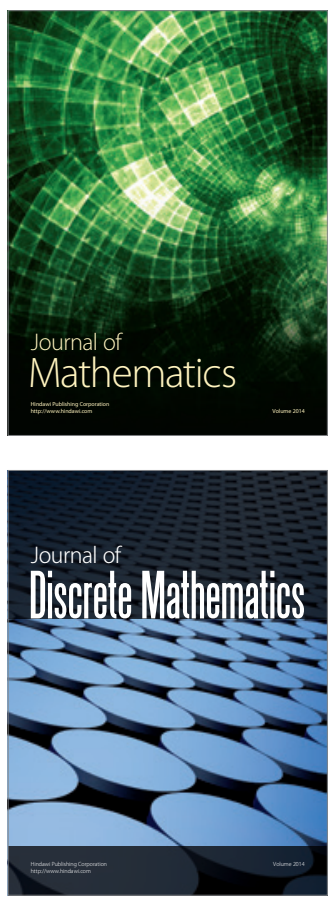

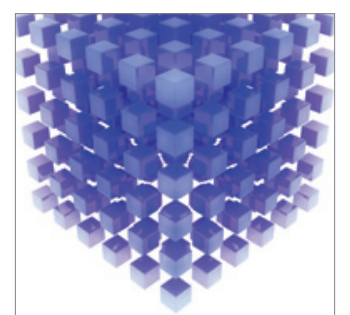

Mathematical Problems in Engineering
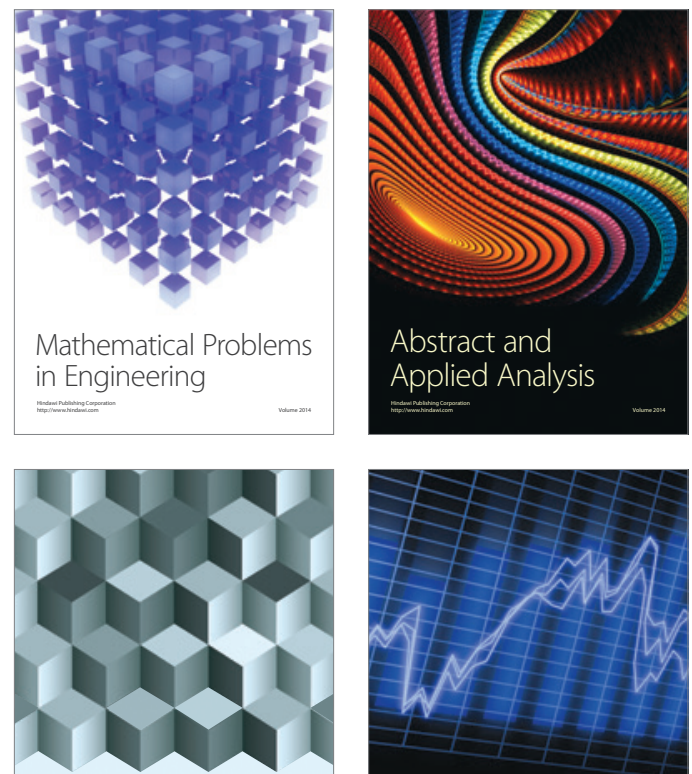

Journal of

Function Spaces

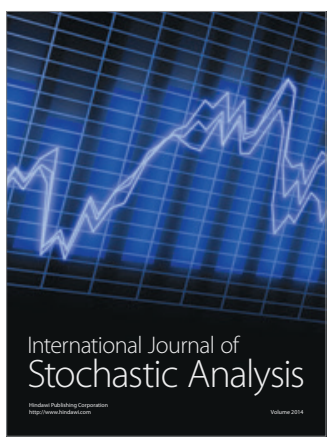

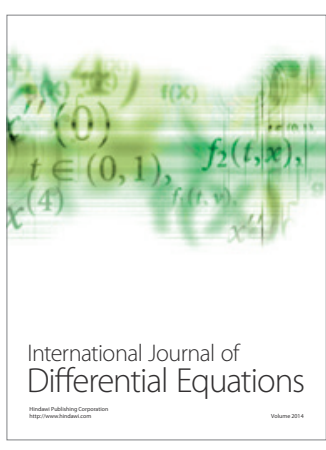
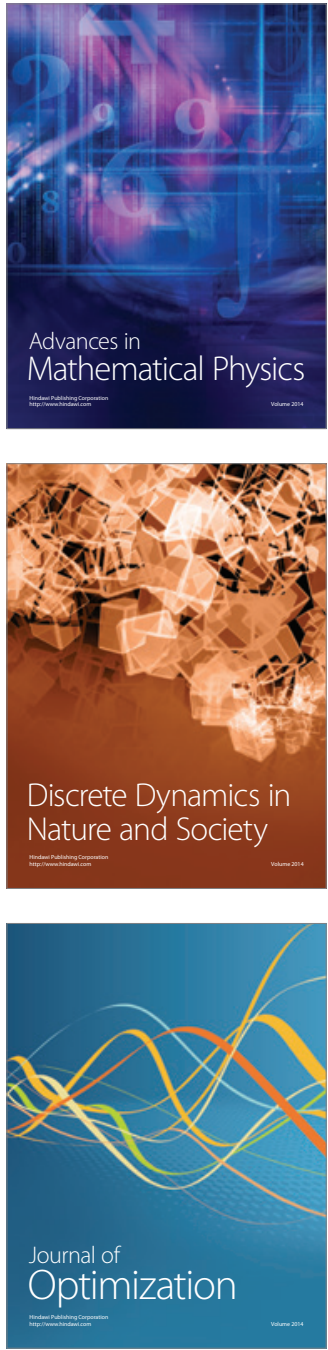